\title{
Highly Stable Nanoparticle Supercrystals Formed by Aldol Reaction in Conjunction with Slow Solvent Evaporation
}

\section{Jiwhan Kim}

Korea Advanced Institute of Science and Technology

Jahar Dey

Korea Advanced Institute of Science and Technology

Aminah Umar

Korea Advanced Institute of Science and Technology

Jae-Min Ha

Department of Nuclear and Quantum Engineering, Korea Advanced Institute of Science and Technology

\section{Sang-Jo Lee}

Korea Advanced Institute of Science and Technology

Sung-Min Choi ( $\nabla$ sungmin@kaist.ac.kr)

Korea Advanced Institute of Science and Technology

\section{Article}

Keywords: Highly Stable, Nanoparticle, Supercrystals, Aldol Reaction, Slow Solvent Evaporation

Posted Date: September 10th, 2020

DOl: https://doi.org/10.21203/rs.3.rs-68341/v1

License: (1) This work is licensed under a Creative Commons Attribution 4.0 International License.

Read Full License 


\title{
Highly Stable Nanoparticle Supercrystals Formed by Aldol Reaction in Conjunction with Slow Solvent Evaporation
}

\author{
Jiwhan Kim $^{1 *}$, Jahar Dey ${ }^{1 *}$, Aminah Umar ${ }^{1}$, Jae-Min Ha ${ }^{1}$, Sang-Jo Lee ${ }^{1}$, and Sung-Min Choi ${ }^{1}$ \\ ${ }^{1}$ Department of Nuclear and Quantum Engineering, Korea Advanced Institute of Science and \\ Technology, Daejeon 34141, Republic of Korea
}

"These authors contributed equally to this work.

Correspondence and request for materials should be addressed to S.-M. Choi (sungmin@kaist.ac.kr)

\begin{abstract}
The nanoparticle supercrystals (NPSCs) have been of great interests for their collective emergent properties. While various NPSCs have been successfully fabricated using intermolecular forces, the limited structural stability of NPSCs due to the weak nature of the intermolecular forces still remains a major hurdle for practical applications. Herein, we report a new method to fabricate highly stable three-dimensional NPSCs by using aldol reaction, a model covalent bond forming reaction, in conjunction with slow solvent evaporation. Gold nanoparticles functionalized with thiol poly-ethylene glycol formyl are linked to each other by carbon-carbon covalent bonds formed by aldol reaction as the particle dispersion in aqueous $\mathrm{NaOH}$ solution is slowly evaporated, resulting in highly faceted three-dimensional NPSCs. Assynthesized NPSCs show excellent structural stability in solvents of different polartities as well as the dried condition and at temperature up to $160^{\circ} \mathrm{C}$, which is far superior to NPSCs stabilized by intermolecular forces such as hydrogen bonding and van der Waals interactions. The new covalent bonding appraoch opens up new opportunities in the synthesis of NPSCs and their applications.
\end{abstract}




\section{Introduction}

Nanoparticle supercrystals have been of great interest for their new emergent properties including plasmonic ${ }^{1-3}$, optical ${ }^{4-6}$, electrical ${ }^{7,8}$, and magnetic ${ }^{9,10}$ properties which cannot be achieved by individual nanoparticles. The emergent properties of nanoparticle supercrystals originate from collective interaction between nanoparticles and are determined by the crystal symmetry $^{11,12}$ and lattice parameters ${ }^{13}$ as well as composition ${ }^{14}$. Various intermolecular interactions such as hydrogen bonding ${ }^{15,16}$ and van der Waals interaction ${ }^{17,18}$ have been successfully utilized to synthesize and stabilize NPSCs of different symmetries and lattice parameters. However, the limited structural stability of NPSCs fabricated by the relatively weak intermolecular interaction has been a major hurdle for realizing the potential applications. For example, the NPSCs formed by the DNA-mediated method (stabilized by hydrogen bonds) is stable only in saline solution and below DNA melting temperature ${ }^{19}$ and the NPSCs formed by a slow solvent evaporation method (stabilized by van der Waals interaction) are stable only in dried condition ${ }^{20}$.

The intramolecular interactions are much stronger than the intermolecular interactions. Therefore, if intramolecular interactions are used to form and stabilize the NPSC, the structural stability of NPSC would be significantly enhanced. Covalent bonding interactions are one of the most representative intramolecular interactions and a broad spectrum of covalent bonding interactions are available and have been extensively utilized to form small molecules and covalent organic framework ${ }^{21}$. Therefore, the covalent bonds can be an excellent candidate for forming new NPSCs with significantly enhanced structural stability. In addition to the wellestablished covalent bonds chemistry and its versatility, the relatively much cheaper price of molecules for covalent bond forming reaction than the DNA counterpart would be a great advantage for practical applications. In the past few years, the molecular cross-linking has been 
(a)
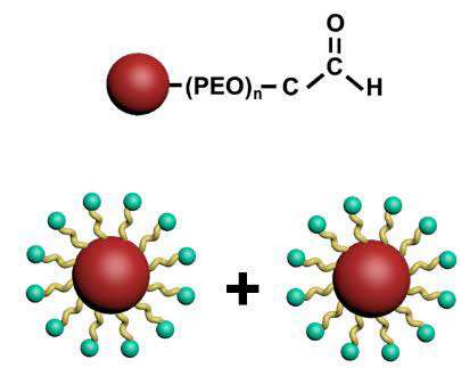

$\stackrel{\mathrm{NaOH}}{\longrightarrow}$

$\mathrm{Au}-\mathrm{CHO}$

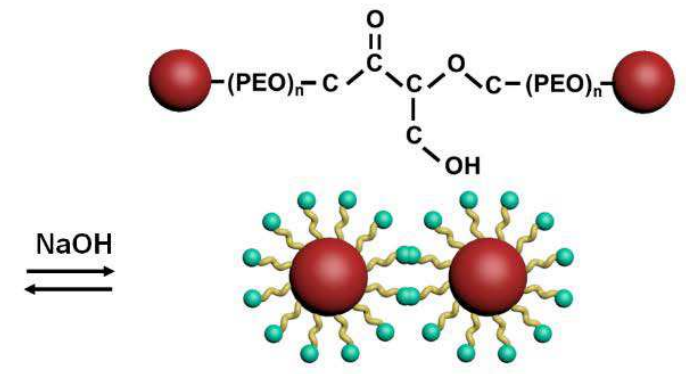

Covalently Bonded Au NPs

(b)

$$
\mathrm{Au}-\mathrm{CHO}
$$

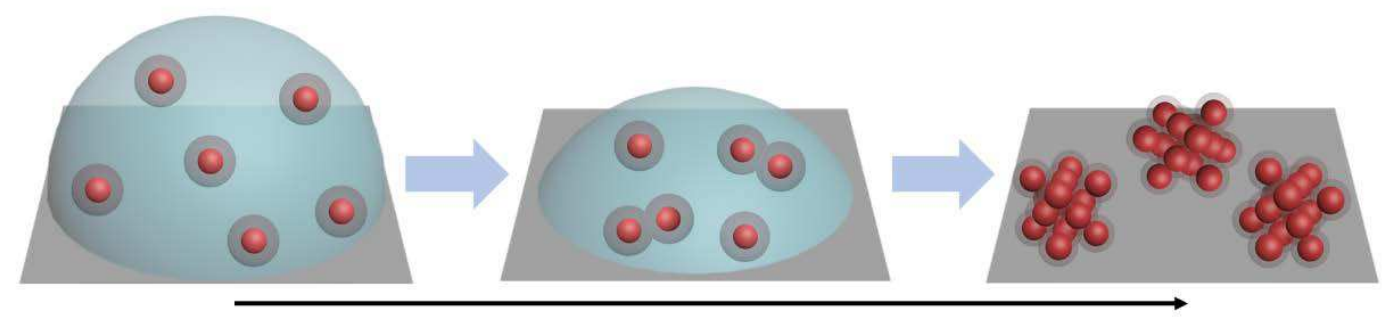

Covalent bonding \& Solvent evaporation

Figure 1. (a) Schematics for aldol reaction between formyl groups functionalized on $\mathrm{Au}$ nanoparticles (Au-CHO) in the presence of $\mathrm{NaOH}$. (b) Schematic for the formation of NPSCs by using covalent bonds interaction in conjunction with slow solvent evaporation.

used to increase the stability of pre-formed NPSCs by thermal treatment ${ }^{22}$ or ultraviolet irradiation $^{23,24}$. However, the utilization of covalent bonding interaction as a main driving force in the formation of NPSCs has not been reported yet.

Here, we report a new method to fabricate highly stable NPSCs by using covalent bonding interaction in conjunction with the slow solvent evaporation process. Gold nanoparticles (Au NPs) functionalized with thiol poly-ethylene glycol formyl (HS-PEG-CHO) are dispersed in aqueous $\mathrm{NaOH}$ solution and slowly evaporated on a substrate, resulting in NPSCs stabilized with covalent bonds, as illustrated in Figure 1. In this method, the aldol reaction is used as a model covalent bond forming reaction in which formyl groups $(-\mathrm{CHO})$ in the presence of a base react each other to form $\beta$-hydroxy carbonyl (called aldol group) compounds. The NPSCs fabricated with this method are highly stable in solvents of different polarity ranging from water to toluene and at temperature up to $160{ }^{\circ} \mathrm{C}$. As-synthesized NPSCs show superior solvent and 
thermal stability than NPSCs fabricated by previous methods and provide new opportunities for a wide range of potential applications.

\section{Results and Discussions}

\section{Fabrication of Gold Nanoparticle Supercrystal}

Highly monodisperse nanoparticles of two different shapes, spherical Au NPs with a diameter of $26.3 \pm 1.1 \mathrm{~nm}$ (Figure S2) and octahedral Au NPs with an edge length of $26.8 \pm$ $1.2 \mathrm{~nm}$ (Figure S3) representing isotropic and anisotropic building blocks, respectively, are synthesized by a seed-mediated method. The functionalization of Au NPs with HS-PEG-CHO is taken place in two steps. First, the Au NPs stabilized with cetylpyridinium chloride are mixed with $1 \mathrm{kDa}$ thiol poly-ethylene glycol hydroxyl (HS-PEG-OH) in aqueous solution, resulting in Au NPs functionalized with HS-PEG-OH. Second, the Au NPs functionalized with HS-PEG$\mathrm{OH}$ (denoted as $\mathrm{Au}-\mathrm{OH}$ ) are converted to the Au NPs functionalized with HS-PEG-CHO (denoted as $\mathrm{Au}-\mathrm{CHO}$ ) by adding pyridinium chlorochromate into the solution. The resulting functionalized Au NPs are washed with water twice and the conversion of - $\mathrm{OH}$ group to - $\mathrm{CHO}$ groups are confirmed by Fourier transform-infrared (FT-IR) spectrometer measurement (Figure S4). The FT-IR data shows that the $-\mathrm{OH}$ peak disappears with addition of pyridinium chlorochromate, indicating that most of $\mathrm{Au}-\mathrm{OH}$ were converted to Au-CHO. The thermogravimetric analysis (TGA) measurement of spherical Au NPs functionalized with HS-

PEG-CHO shows that the number of ligands per unit area is $8.4 \mathrm{~nm}^{-2}$ (Figure S5). The Au$\mathrm{CHO}$ particles are dispersed in water at $136 \mathrm{mg} \mathrm{ml}^{-1} .10 \mu \mathrm{L}$ of the dispersion is mixed with 5 $\mu \mathrm{L}$ of $0.1 \mathrm{M} \mathrm{NaOH}$ (aq.), and then the mixture is allowed to evaporate on a silicon wafer placed inside a petri dish (with $2 \mathrm{ml}$ of water added) for 5 days at room temperature. All the procedures described above are performed for both spherical and octahedral Au NPs, respectively. 


\section{With $\mathrm{NaOH}$}
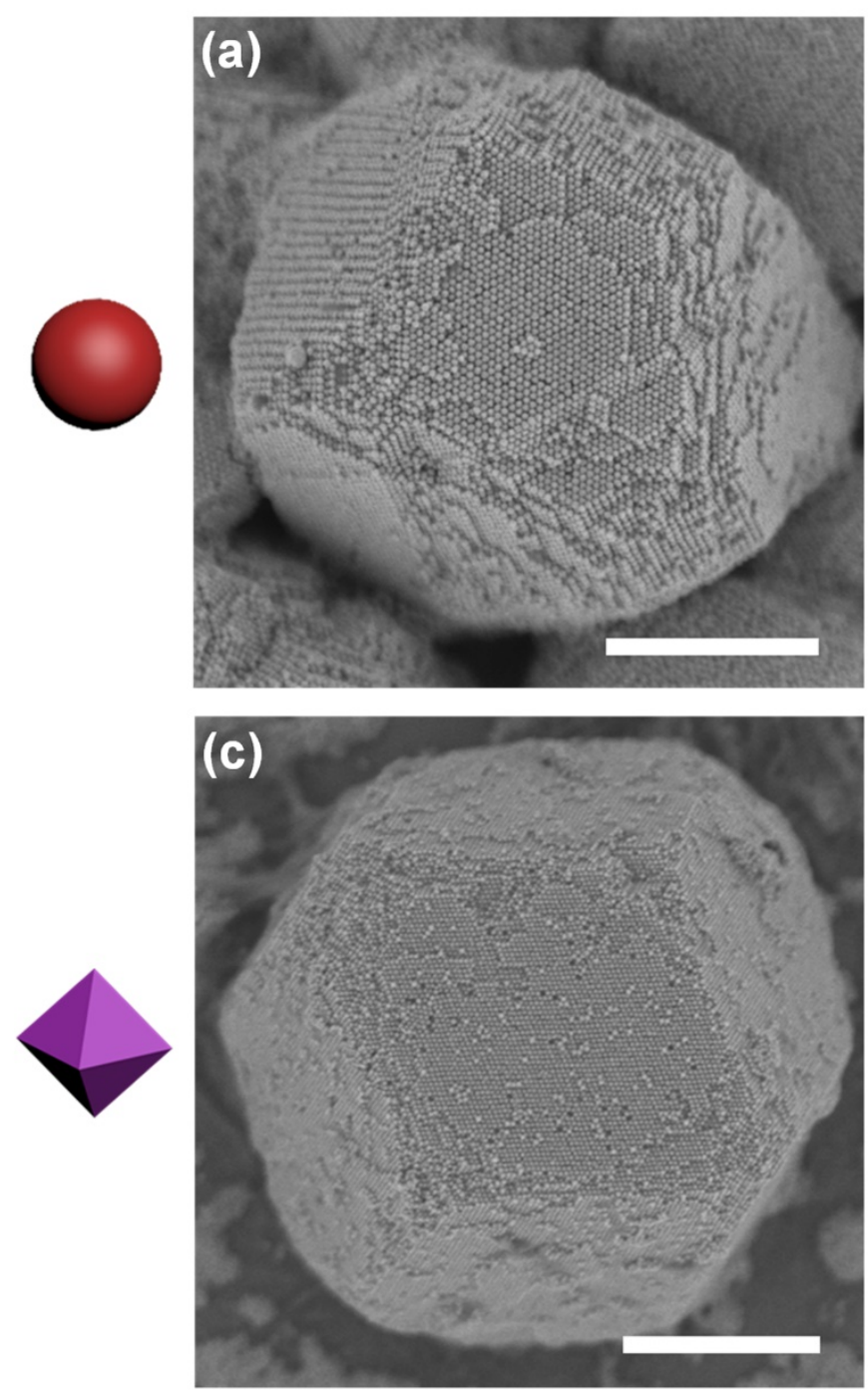

Without $\mathrm{NaOH}$
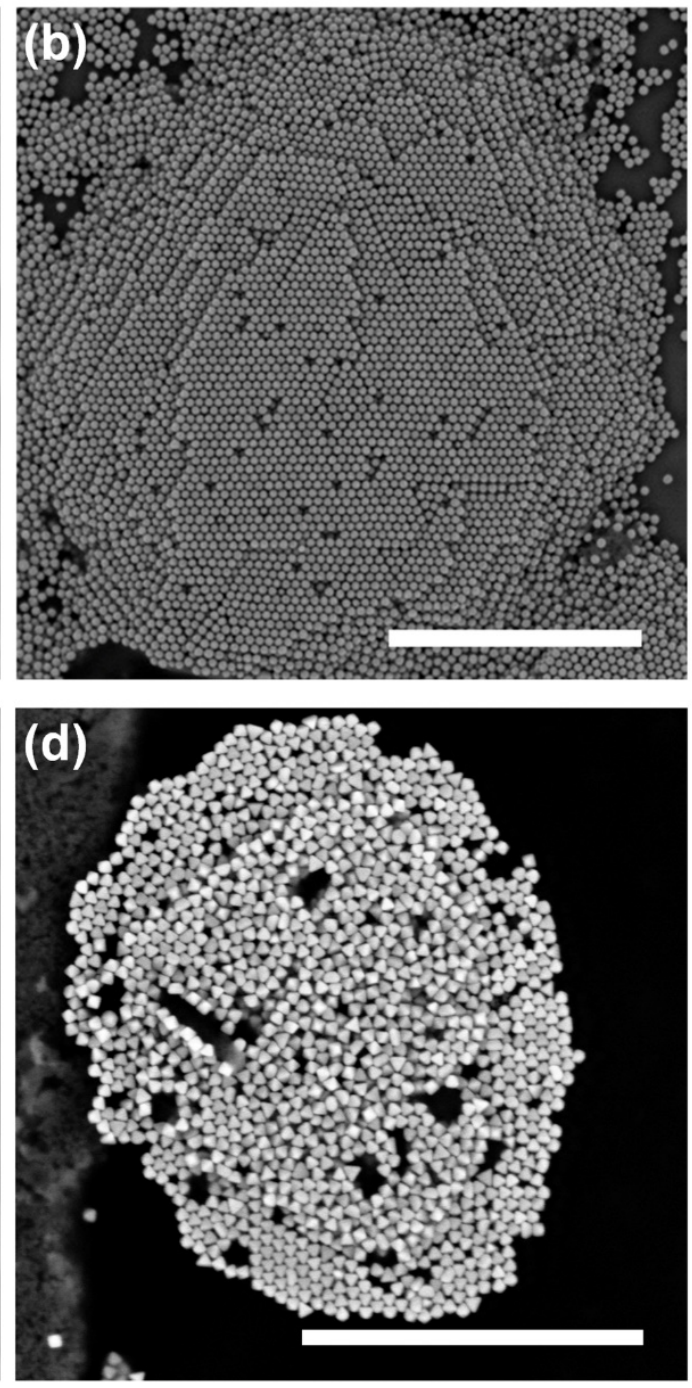

Figure 2. SEM images of NPSCs formed by spherical (a, b) and octahedral (c, d) Au NPs with (a, c) and without $(b, d) \mathrm{NaOH}$. The scale bars are $1 \mu \mathrm{m}$.

\section{Characterization of Au NPSCs: Structures and Covalent Bonding}

To investigate the morphology of NPSCs, the scanning electron microscopy (SEM) measurements of the aggregates on silicon wafers after 5 days of evaporation were performed. The SEM images show that highly faceted micrometer-sized three-dimensional NPSCs are formed for both spherical and octahedral Au NPs (Figure 2a and 2c). The NPSCs formed with spherical particles also show truncated octahedral shape and the NPSCs formed with octahedral 
particles show rhombic dodecahedral shape. It should be noted that both of the NSPC shapes are Wulff polyhedral which are obtained when the surface energy is minimized ${ }^{25}$. To understand the effects of aldol reaction on the formation of NPSCs, the aggregates formed by slow evaporation without aldol reaction are prepared and compared. For this purpose, the Au NPs functionalized with HS-PEG-CHO are slowly evaporated at the same condition as described above without adding $\mathrm{NaOH}(5 \mu \mathrm{L}$ of water is used instead of $5 \mu \mathrm{L}$ of $0.1 \mathrm{M} \mathrm{NaOH}$ (aq.)). The SEM measurements show that spherical and octahedral Au NPs form two-dimensional NPSCs (with one or a few layers of hexagonally packed particles), which is typical for NPSCs formed by slow solvent evaporation (Figure $\mathbf{2 b}$ and $\mathbf{2 d}$ ) $^{26}$. This is in stark contrast with the morphology of NPSCs formed in the presence of $\mathrm{NaOH}$. The differences clearly indicate that the aldol reaction plays a key role in the formation of highly faceted three-dimensional NPSCs.

To confirm the formation of covalent bonds in the NPSCs prepared with addition of $\mathrm{NaOH}$, the FT-IR spectroscopy measurement is performed. The FT-IR spectrum of Au NPs functionalized with HS-PEG-CHO shows peaks corresponding to the formyl group (-CHO), a peak for the $\mathrm{C}=\mathrm{O}$ bonds at $1710 \mathrm{~cm}^{-1}$ and two peaks for the $\mathrm{C}-\mathrm{H}$ bonds at $2850 \mathrm{~cm}^{-1}$ and 2930 $\mathrm{cm}^{-1}$ (Figure 3a) ${ }^{27}$. The FT-IR spectrum of the NPSCs prepared with addition of $\mathrm{NaOH}$ shows a broad and strong peak at around $3250 \mathrm{~cm}^{-1}$ correspondings to the $\mathrm{O}-\mathrm{H}$ bonds of aldol group ${ }^{27}$, which is absent in the spectrum of Au NPs functionalized with HS-PEG-CHO. This clearly confirms the formation of covalent bonds between Au NPs by an aldol reaction. The peaks corresponding to the $\mathrm{C}=\mathrm{O}$ bonds (which are present in both the formyl and aldol groups) and $\mathrm{C}-\mathrm{H}$ bonds (which are present in the formyl group) are also shown in the spectrum. This indicates that a small fraction of the formyl group remains without being used for the aldol reaction. 
(a)

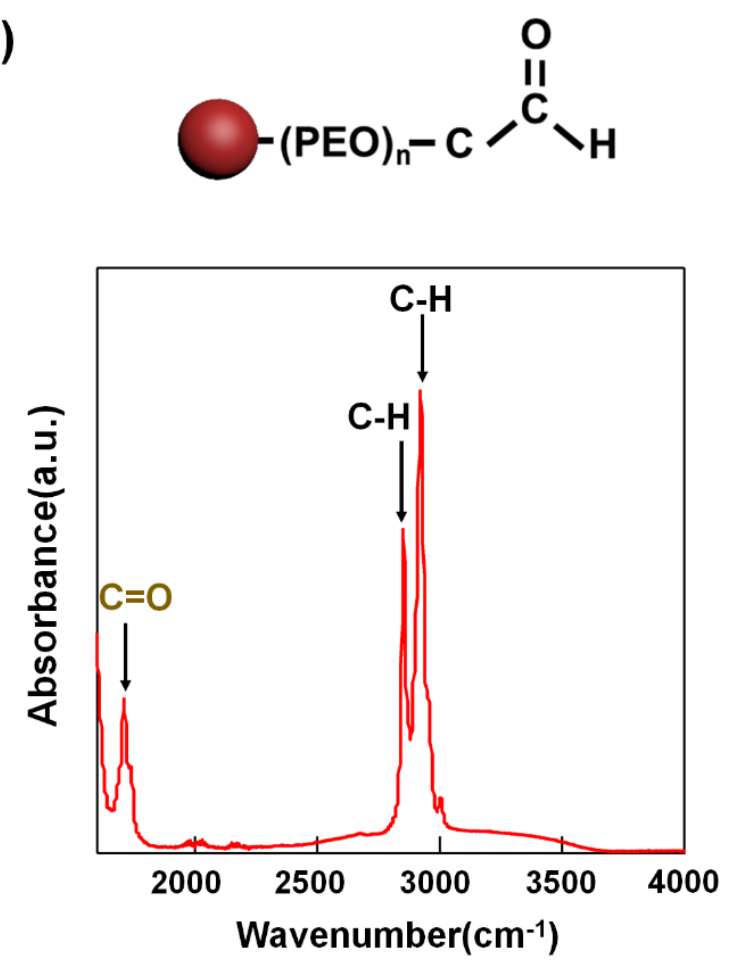

(b)
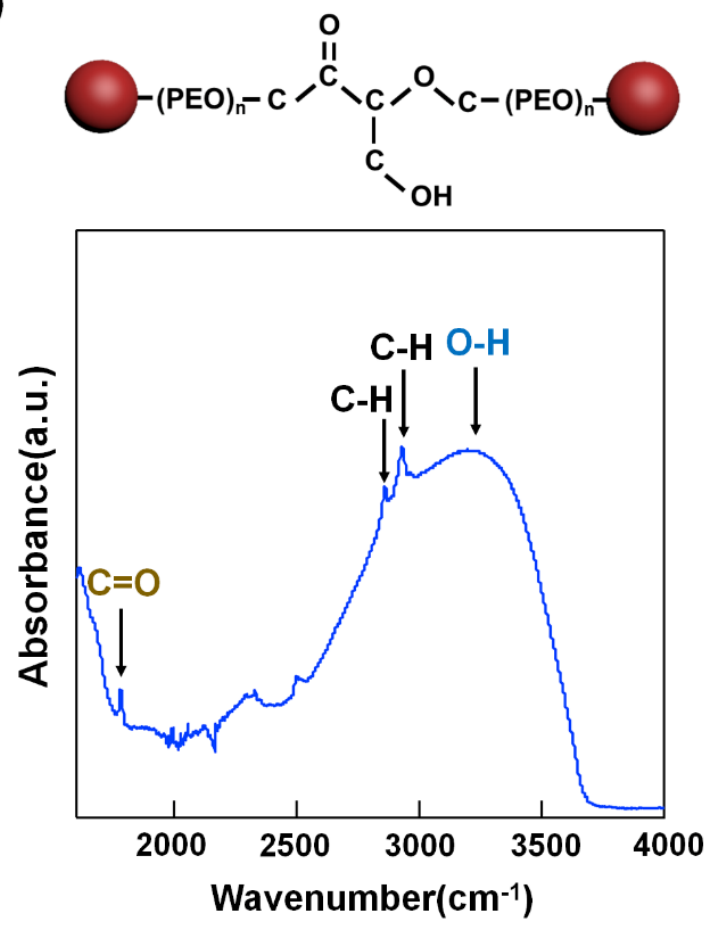

Figure 3. (a) FT-IR spectrum of spherical Au NP functionalized with HS-PEG-CHO and (b) FT-IR spectrum of spherical Au NPSCs fabricated by using aldol reaction in conjunction with slow solvent evaporation.

To investigate the structure of NPSCs, small-angle x-ray scattering (SAXS) measurements are performed for the Au NPSCs formed by aldol reaction in conjunction with slow solvent evaporation. The SAXS intensity of NPSCs made of spherical Au NPs shows distinct peaks, which can be indexed with the face-centered cubic (fcc) symmetry with the lattice parameters of $39.7 \mathrm{~nm}$. The presence of high order peaks indicates that NPSCs are highly ordered. The fcc symmetry has the highest packing density of $74 \%$ for spherical particles and the highest coordination number of 12 . Therefore, for the spherical particles which bind each other through an aldol reaction, the fcc symmetry provides the minimum system energy both entropically and enthalpically ${ }^{28}$. The distance between the nearest neighboring spherical Au NPs estimated from the lattice parameter is $28.1 \mathrm{~nm}$. Considering that the diameter of spherical Au NPs is $26.3 \mathrm{~nm}$, the gap between neighboring particles filled with covalently connecting molecules is $1.8 \mathrm{~nm}$. The domain size estimated from the (111) peak using the Scherrer's equation is ca. $1.5 \mu \mathrm{m}$, 


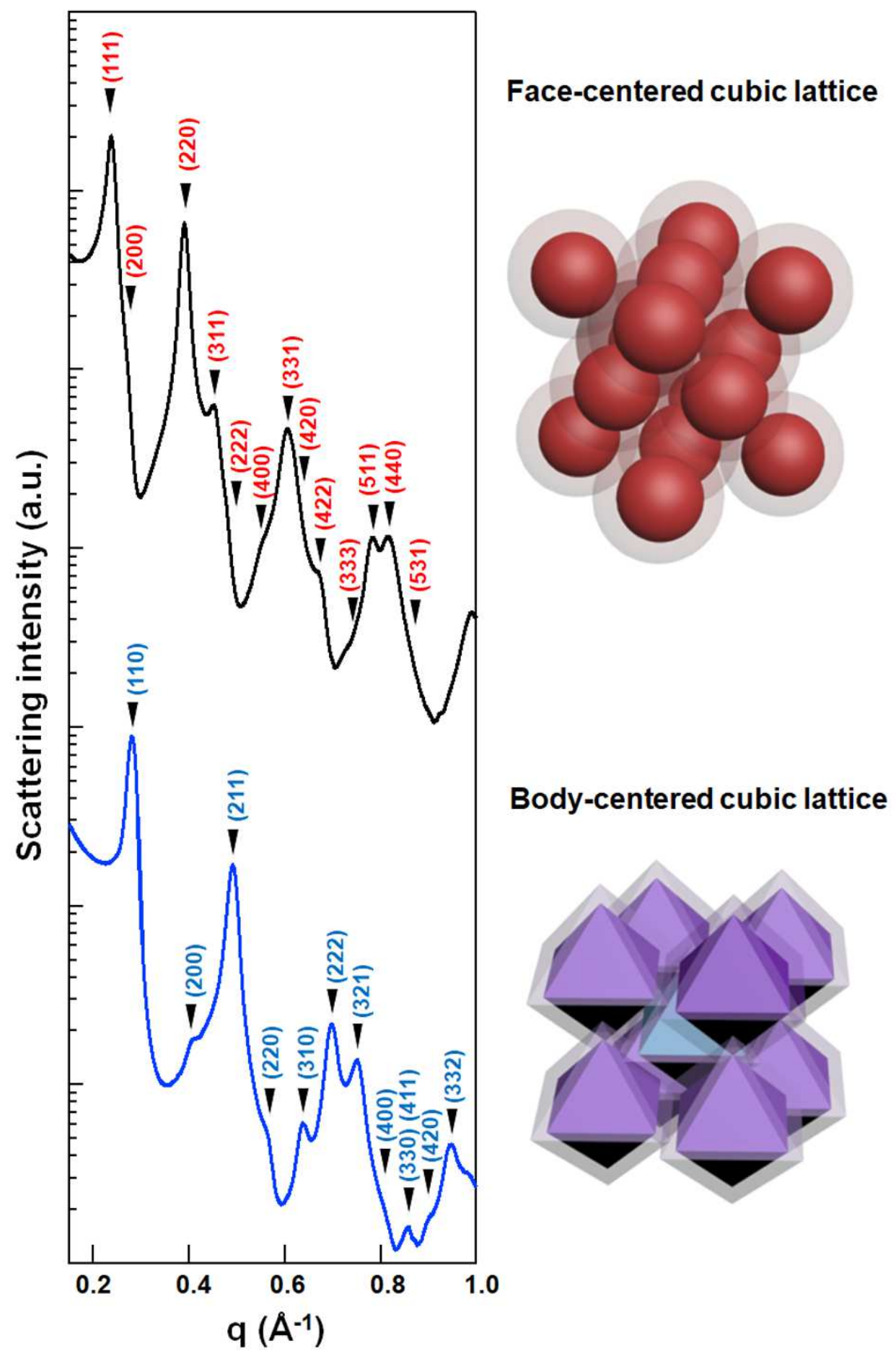

Figure 4. SAXS intensities of spherical (black) and octahedral (blue) Au NPSCs formed by aldol reaction in conjunction with slow solvent evaporation. SAXS intensity of spherical $\mathrm{Au}$ NPSCs is indexed with the fcc symmetry and SAXS intensity of octahedral Au NPSCs is indexed with the bcc symmetry. SAXS intensities are shifted vertically for visual clarity.

which is consistent with the size of NPSCs estimated from SEM images. This suggests that the NPSCs are mostly single crystal. 
The SAXS intensity of NPSCs made of octahedral Au NPs shows distinct high order peaks up to more than $10^{\text {th }}$ order, indicating that highly ordered NPSCs are formed. All the peaks can be indexed by the body-centered cubic (bcc) symmetry with a lattice parameter of $31.5 \mathrm{~nm}$. This is consistent with previous Monte Carlo simulation, free energy calculation, and experimental studies which show that the bcc phase is most stable for octahedral particles ${ }^{29-31}$. Using the measured lattice parameter and the size of octahedral particle, the particle packing density, and the orientation of octahedral particles relative to the axis of bcc unit cell are estimated. The particle packing density is ca. $70.5 \%$ and the line connecting two opposite vertices of the octahedral particle is tilted ca. $12.1^{\circ}$ from the c-axis of a unit cell. In this estimation, the molecular shell of $0.9 \mathrm{~nm}$ thickness on octahedral Au NPs is included in the volume of octahedral particles. The domain size estimated from the (110) peak using the Scherrer's equation is ca. $1.4 \mu \mathrm{m}$. This is consistent with the SEM measurements which predominantly show NPSCs of 1-2 $\mu \mathrm{m}$ size. This also suggests that the NPSCs are mostly single crystal.

The formation of three-dimensional NPSCs by aldol reaction in conjunction with slow solvent evaporation can be attributed to the nucleation in the solution bulk and the reversibility of aldol reaction. Considering that the slow solvent evaporation typically induces a thin film of NPSCs by nucleation and growth at the air-liquid interface, ${ }^{26}$ the formation of threedimensional NPSCs suggests that nucleation and growth may have occured mainly in the solution bulk by aldol reaction between functionalized Au NPs. To form highly ordered NPSCs, particles should be allowed to re-adjust their positions or orientations after clustering to minimize the free energy. In this method, this is provided by the reversibility of aldol reaction, i.e. the retro-aldol reaction in the presence of $\mathrm{NaOH}$ temporally decomposes the aldol group back to the initial formyl groups ${ }^{32}$. 


\section{Structural Stability of Au NPSCs Formed by Aldol Reaction}

The carbon-carbon covalent bonds formed by the aldol reaction is highly stable in a wide range of solvents. Therefore, the Au NPSCs fabricated by aldol reaction in conjunction with slow solvent evaporation, in which Au NPs are linked to each other by the carbon-carbon covalent bonds, should have high solvent stability. The structural stability of spherical and octahedral Au NPSCs in solvents of different polarities is investigated by SAXS and SEM measurements (Figure 5). For this purpose, the Au NPSCs are immersed in water, ethanol, and toluene for 24 hours, respectively, and dried before the measurements. All the SAXS intensities of Au NPSCs treated with different solvents show distinct peaks which are essentially the same as the scattering intensities of the samples without immersion in the solvent. This indicates that the lattice symmetry and the degree of ordering of spherical and octahedral Au NPSCs are wellmaintained in solvents of different polarities. The SEM images further confirm the excellent solvent stability of Au NPSCs, showing highly faceted supercrystals. This is in stark contrast to NPSCs fabricated by the slow solvent evaporation which are destroyed once it is placed in solvent, and NPSCs fabricated by the DNA-mediated method which is stable only in saline aqueous solution below the DNA melting temperature.

The thermal stability of spherical and octahedral Au NPSCs formed by aldol reaction in conjunction with slow solvent evaporation is investigated by SAXS measurements at different temperatures ranging from $20^{\circ} \mathrm{C}$ to $180{ }^{\circ} \mathrm{C}$ with $20{ }^{\circ} \mathrm{C}$ step (Figure S6). At each temperature, the samples are allowed for 30 minutes before the measurement. The distinct peaks in the SAXS intensities are well-maintained up to $160{ }^{\circ} \mathrm{C}$ for both spherical and octahedral Au NPSCs. This is superior than the NPSCs fabricated by the DNA-mediated method which become destabilized above the DNA melting temperature which is in the range of $30-45^{\circ} \mathrm{C}$ depending the number of base pairs used. 

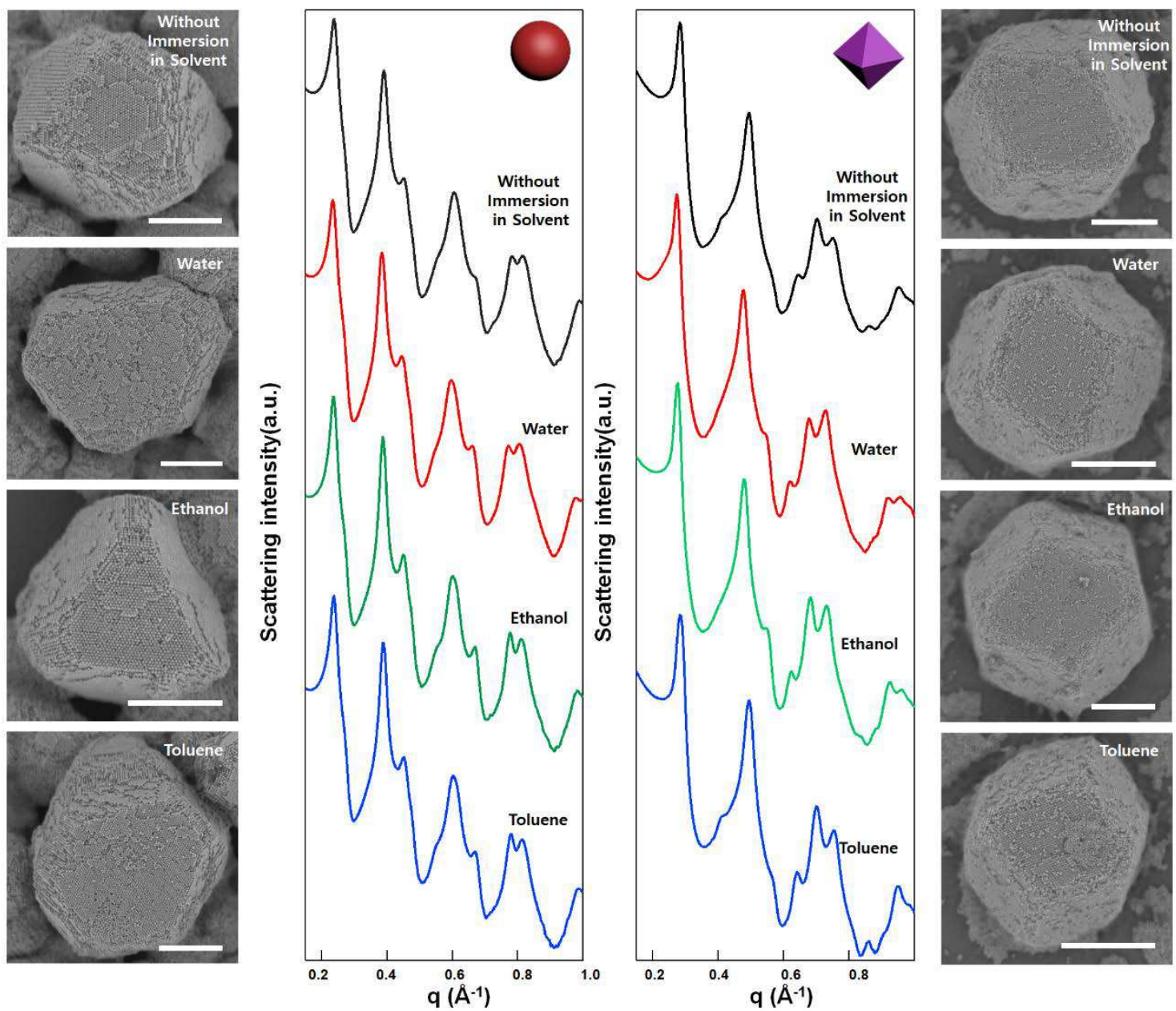

Figure 5. SAXS intensities and SEM images of spherical (left) and octahedral (right) $\mathrm{Au}$ NPSCs formed by aldol reaction after 24 hours of immersion in water, ethanol, and toluene. SAXS intensities and SEM images of Au NPSCs without immersion in solvents are also presented for comparison. SAXS intensities are shifted vertically for visual clarity and the scale bars in SEM images are $1 \mu \mathrm{m}$.

In summary, we have successfully developed a new method to fabricate highly stable and highly ordered three-dimensional NPSCs by using aldol reaction, a covalent bond forming reaction, in conjunction with slow solvent evaporation and demonstrated the method for isotropic and anisotropic nanoparticles. The NPSCs formed and stabilized by aldol reaction show excellent structural stability in a broad spectrum of solvents ranging from water to toluene and at temperature up to $160{ }^{\circ} \mathrm{C}$, which is far superior to NPSCs stabilized by intermolecular interactions such as hydrogen bonds and van der Waals interactions. The new covalent bonds approach to form stable NPSCs may utilize a wide spectrum of covalent bond forming reactions, 
making the method highly versatile, and the superior structural stability provided by this approach is an important step forward for the practical applications of NPSCs in working environments with different temperature and solvent conditions.

\section{Methods}

Materials. Cetyltrimethylammonium bromide (CTAB, $\geq 98 \%$ ), 4-vinyl benzoic acid (VBA, $\geq 97 \%$ ), gold (III) chloride hydrate $\left(\mathrm{HAuCl}_{4} \cdot \mathrm{H}_{2} \mathrm{O}, \geq 99.9 \%\right.$ ), gold (III) chloride trihydrate $\left(\mathrm{HAuCl}_{4} \cdot 3 \mathrm{H}_{2} \mathrm{O}, \geq 99.9 \%\right)$, silver nitrate $\left(\mathrm{AgNO}_{3}, \geq 99 \%\right)$, hydrochloric acid $(\mathrm{HCl}, 37 \%)$, nitric acid $\left(\mathrm{HNO}_{3}, \geq 65 \%\right.$ ), anion exchange resin (Dowex Monosphere 550A UPW), ascorbic acid $\left(\mathrm{C}_{6} \mathrm{H}_{8} \mathrm{O}_{6}, \geq 65 \%\right)$, cetylpyridinium chloride $(\mathrm{CPC}, \geq 99 \%)$, pyridinium chlorochromate (PCC, $\geq$ $98 \%$ ), sodium hydroxide $(\mathrm{NaOH}, \geq 98 \%)$ were purchased from Sigma-Aldrich and used as received. Cetyltrimethylammonium 4-vinylbenzoate (CTAVB) was synthesized using a previously reported method ${ }^{33,34}$. Thiol poly-ethylene glycol hydroxyl (HS-PEG-OH, molecular weight $1 \mathrm{k} \mathrm{Da}$ ) was purchased from Creative PEGWorks and used as obtained. $\mathrm{H}_{2} \mathrm{O}$ was purified using a Millipore Direct Q system (electrical resistivity of 18.2 M $\Omega$ ). All glassware was cleaned by aqua regia solution $\left(\mathrm{HCl}: \mathrm{HNO}_{3}\right.$ in 3: 1 by molar ratio). Silicon wafer was washed by piranha solution $\left(\mathrm{H}_{2} \mathrm{SO}_{4}: \mathrm{H}_{2} \mathrm{O}_{2}\right.$ in 3:1 by molar ratio) before use

\section{Synthesis of $13 \mathrm{~nm}$ single-crystalline spherical Au nanoparticles which are used as seeds.}

The single-crystalline Au nanoparticles (NPs), which are used as seeds for the synthesis of other Au NPs, are synthesized by using a method developed by our group ${ }^{35} .81 \mathrm{mg}$ of CTAVB is dissolved in $25 \mathrm{~mL}$ of acidic aqueous solution (0.01 M HCl, $\mathrm{pH}=2) .357 \mu \mathrm{L}$ of $7.0 \mathrm{mM} \mathrm{HAuCl}_{4}$ and $50 \mu \mathrm{L}$ of $\mathrm{AgNO}_{3}$ were added to the mixture at $30{ }^{\circ} \mathrm{C}$ under stirring, resulting in concentrations of CTAVB, $\mathrm{HAuCl}_{4}$, and $\mathrm{AgNO}_{3}$ of $7.5 \mathrm{mM}, 0.1 \mathrm{mM}$, and $0.002 \mathrm{mM}$ respectively. The resulting mixture was then stirred for 1 hour at $30^{\circ} \mathrm{C}$ and then kept in a water 
bath maintained at $42{ }^{\circ} \mathrm{C}$ for 72 hours. The samples were centrifuged and washed with water, which was repeated twice. The Field-emission transmission electron microscope (FE-TEM, Tecani G2 F30) measurements show that monodisperse spherical Au NPs are formed with a diameter of $13.2 \mathrm{~nm}$ and a polydispersity of $5.1 \%$ (Figure S1).

Synthesis of $26 \mathrm{~nm}$ spherical Au NPs by a seed-mediated method. $26 \mathrm{~nm}$ spherical Au NPs were synthesized by a seed-mediated method. A growth solution was prepared by adding 0.1 $\mathrm{mL}$ of $10 \mathrm{mM} \mathrm{HAuCl}_{4}$ aqueous solution and $30 \mu \mathrm{L}$ of freshly prepared $100 \mathrm{mM}$ ascorbic acid into $5 \mathrm{~mL}$ of $100 \mathrm{mM} \mathrm{CPC}$ solution. $100 \mu \mathrm{L}$ of $0.17 \mathrm{mg} \mathrm{mL}^{-1} \mathrm{Au}$ seed solution (singlecrystalline $13.2 \mathrm{~nm}$ Au NPs) was added into the growth solution and kept stirring for 1 hour, during which the temperature was kept at $30{ }^{\circ} \mathrm{C}$. The FE-TEM measurements (Tecnai G2 F30) show that monodisperse spherical Au NPs are formed with a diameter of $26.3 \mathrm{~nm}$ and a polydispersity of $4.1 \%$ (Figure S2).

Synthesis of octahedral Au NPs by a seed-mediated method. $26 \mathrm{~nm}$ octahedral Au NPs were synthesized by a seed-mediated method ${ }^{36}$. A growth solution was prepared by adding $0.1 \mathrm{~mL}$ of $10 \mathrm{mM} \mathrm{HAuCl}_{4}$ aqueous solution and $13 \mu \mathrm{L}$ of freshly prepared $100 \mathrm{mM}$ ascorbic acid into $5 \mathrm{~mL}$ of $100 \mathrm{mM}$ CPC solution. $100 \mu \mathrm{L}$ of $0.17 \mathrm{mg} \mathrm{mL}^{-1}$ Au seed solution (single-crystalline 13.2 $\mathrm{nm} \mathrm{Au} \mathrm{NPs)} \mathrm{was} \mathrm{added} \mathrm{into} \mathrm{the} \mathrm{growth} \mathrm{solution} \mathrm{and} \mathrm{kept} \mathrm{stirring} \mathrm{for} 1$ hour, during which the temperature was kept at $30{ }^{\circ} \mathrm{C}$. The SEM measurements (Hitachi Su8230) show that monodisperse octahedral Au NPs are formed with an edge length of $26.8 \mathrm{~nm}$ and a polydispersity of $4.4 \%$ (Figure S3).

Functionalization of spherical and octahedral Au NPs with HS-PEG-CHO. $0.05 \mathrm{~mL}$ of 0.05 M HS-PEG-OH (1 kDa) aqueous solution was added into $10 \mathrm{~mL}$ aqueous solution of CPC capped Au NPs (0.14 mg mL $\mathrm{m}^{-1}$, spherical Au NPs of $26.3 \mathrm{~nm}$ and octahedral Au NPs with an 
edge length of $26.8 \mathrm{~nm}$, respectively). The mixture was kept undisturbed for 12 hours, resulting in $\mathrm{Au}$ NPs functionalized with HS-PEG-OH (denoted as Au-OH). To convert the hydroxyl group (-OH) to formyl group (-CHO), $0.05 \mathrm{~mL}$ of $50 \mathrm{mM}$ PCC aqueous solution was added to the mixture and kept undisturbed for 4 hours, resulting in Au NPs functionalized with HS-PEG$\mathrm{CHO}$ (denoted as $\mathrm{Au}-\mathrm{CHO}$ ). The samples were centrifuged and washed with water, which was repeated twice.

To confirm the conversion of -OH group to - $\mathrm{CHO}$ group functionalized on Au NPs, Fouriertransform infrared (FT-IR, Nicolet iS50) measurements were performed (Figure S4). While the FT-IR spectrum of Au-OH shows a broad and strong peak at around $3250 \mathrm{~cm}^{-1}$ correspondings to the O-H bonds, the FT-IR spectrum of Au-CHO (prepared by adding PCC into the Au-OH solution) shows a peak at $1710 \mathrm{~cm}^{-1}$ correspondings to the $\mathrm{C}=\mathrm{O}$ bonds and two peaks at 2850 $\mathrm{cm}^{-1}$ and $2930 \mathrm{~cm}^{-1}$ correspondings to the $\mathrm{C}-\mathrm{H}$ bonds, both of which correspond to the formyl group. The peak corresponding to the $\mathrm{O}-\mathrm{H}$ bonds does not show up in the FT-IR spectrum of $\mathrm{Au}-\mathrm{CHO}$. This clearly confirms the conversion of -OH group to - $\mathrm{CHO}$ group by PCC.

The thermogravimetric analysis (TGA, Libra TG209 F1) was performed for spherical Au NPs functionalized with 1kDa HS-PEG-CHO (Figure 5), from which the areal grafting density of $1 \mathrm{kDa}$ HS-PEG-CHO on Au NPs is determined to be $8.4 \mathrm{~nm}^{-2}$.

\section{Synthesis of Au nanoparticle supercrystals (NPSCs) by aldol reaction in conjunction with} slow solvent evaporation. A mixture of $10 \mu \mathrm{L}$ of $136 \mathrm{mg} \mathrm{ml}^{-1} \mathrm{Au}-\mathrm{CHO}$ aqueous solution and $5 \mu \mathrm{L}$ of $0.1 \mathrm{M} \mathrm{NaOH}$ aqueous solution was dropped on a silicon wafer placed inside a petri dish (with $2 \mathrm{ml}$ of water added) and is allowed to evaporate for 5 days at room temperature. This was performed for spherical and octahedral Au NPs, respectively. 
Small-Angle X-Ray Scattering Measurements. Small-angle X-ray scattering (SAXS) measurements were performed at the beamline 4C of the Pohang Accelerator Laboratory (PAL), Republic of Korea. X-rays with a wavelength $(\lambda)$ of $0.1217 \mathrm{~nm}$ and a wavelength spread $(\Delta \lambda / \lambda)$ of $2 \times 10^{-4}$ delivered by a $\mathrm{Si}(111)$ double crystal monochromator were used. A 2D CCD camera (SX165, Mar USA, Inc. CCD 165) was used to collect scattered X-rays. The sample-to-detector distance of $2 \mathrm{~m}$ was used to cover the $\mathrm{q}$ range of $0.1 \mathrm{~nm}^{-1}<\mathrm{q}<2 \mathrm{~nm}^{-1}$, where $\mathrm{q}=(4 \pi / \lambda) \sin (\theta / 2)$ is the magnitude of the scattering vector and $\theta$ is the scattering angle. The $q$ values were calibrated using silver behenate $\left(\mathrm{AgO}_{2} \mathrm{C}\left(\mathrm{CH}_{2}\right)_{20} \mathrm{CH}_{3}\right)$. The temperature was controlled by using a water circulation bath (Lauda, Germany). All the samples were equilibrated for at least 30 minutes at each temperature before measurement.

\section{References}

1. Ross, M. B., Ku, J. C., Vaccarezza, V. M., Schatz, G. C. \& Mirkin, C. A. Nanoscale form dictates mesoscale function in plasmonic DNA-nanoparticle superlattices. Nat. Nanotechnol. 10, 453-458 (2015).

2. Darby, B. L., Auguié, B., Meyer, M., Pantoja, A. E. \& Le Ru, E. C. Modified optical absorption of molecules on metallic nanoparticles at sub-monolayer coverage. Nat. Photonics 10, 40-45 (2016).

3. Bian, K. et al. Formation of self-assembled gold nanoparticle supercrystals with facetdependent surface plasmonic coupling. Nat. Commun. 9, 1-7 (2018).

4. Chen, Y., Zi, O., Gu, M. \& Cheng, W. Mechanically strong, optically transparent, giant metal superlattice nanomembranes from ultrathin gold nanowires. Adv. Mater. 25, 8085 (2013). 
5. Yang, G., Hu, L., Keiper, T. D., Xiong, P. \& Hallinan, D. T. Gold Nanoparticle Monolayers with Tunable Optical and Electrical Properties. Langmuir 32, 4022-4033 (2016).

6. Sun, L. et al. Polarization-Dependent Optical Response in Anisotropic NanoparticleDNA Superlattices. Nano Lett. 17, 2313-2318 (2017).

7. Kuo, C. H., Yang, Y. C., Gwo, S. \& Huang, M. H. Facet-dependent and Au nanocrystalenhanced electrical and photocatalytic properties of $\mathrm{Au}-\mathrm{Cu}_{2} \mathrm{O}$ core-shell heterostructures. J. Am. Chem. Soc. 133, 1052-1057 (2011).

8. Chiu, C. Y. et al. Surfactant-directed fabrication of supercrystals from the assembly of polyhedral Au-Pd core-shell nanocrystals and their electrical and optical properties. $J$. Am. Chem. Soc. 137, 2265-2275 (2015).

9. Brunner, J. et al. Self-Assembled Magnetite Mesocrystalline Films: Toward Structural Evolution from 2D to 3D Superlattices. Adv. Mater. Interfaces 4, 1-9 (2017).

10. Santos, P. J. \& Macfarlane, R. J. Reinforcing Supramolecular Bonding with Magnetic Dipole Interactions to Assemble Dynamic Nanoparticle Superlattices. J. Am. Chem. Soc. 142, 1170-1174 (2020).

11. Ha, J. M. et al. Micelle-Assisted Formation of Nanoparticle Superlattices and Thermally Reversible Symmetry Transitions. Nano Lett. 19, 2313-2321 (2019).

12. Wang, P. P., Qiao, Q., Zhu, Y. \& Ouyang, M. Colloidal Binary Supracrystals with Tunable Structural Lattices. J. Am. Chem. Soc. 140, (2018).

13. Tian, Y. et al. Lattice engineering through nanoparticle-DNA frameworks. Nat. Mater. 15, 654-661 (2016).

14. Shevchenko, E. V., Talapin, D. V., Kotov, N. A., O’Brien, S. \& Murray, C. B. Structural diversity in binary nanoparticle superlattices. Nature 439, 55-59 (2006).

15. Park, S. Y. et al. DNA-programmable nanoparticle crystallization. Nature 451, 553-556 (2008). 
16. Lewis, D. J., Zornberg, L. Z., Carter, D. J. D. \& Macfarlane, R. J. Single-crystal Winterbottom constructions of nanoparticle superlattices. Nat. Mater. 19, 719-724 (2020).

17. Ye, X. et al. Structural diversity in binary superlattices self-assembled from polymergrafted nanocrystals. Nat. Commun. 6, 1-10 (2015).

18. Huang, M. H. \& Thoka, S. Formation of supercrystals through self-assembly of polyhedral nanocrystals. Nano Today 10, 81-92 (2015).

19. Oh, T., Park, S. S. \& Mirkin, C. A. Stabilization of Colloidal Crystals Engineered with DNA. Adv. Mater. 31, 1-5 (2019).

20. Dalmaschio, C. J. et al. Nanocrystals self-assembled in superlattices directed by the solvent-organic capping interaction. Nanoscale 5, 5602-5610 (2013).

21. Spitler, E. L. \& Dichtel, W. R. Lewis acid-catalysed formation of two-dimensional phthalocyanine covalent organic frameworks. Nat. Chem. 2, 672-677 (2010).

22. Dreyer, A. et al. Organically linked iron oxide nanoparticle supercrystals with exceptional isotropic mechanical properties. Nat. Mater. 15, 522-528 (2016).

23. Yan, N., Liu, X., Zhu, J., Zhu, Y. \& Jiang, W. Well-Ordered Inorganic Nanoparticle Arrays Directed by Block Copolymer Nanosheets. ACS Nano 13, (2019).

24. De Fazio, A. F. et al. Light-Induced Reversible DNA Ligation of Gold Nanoparticle Superlattices. ACS Nano 13, 5771-5777 (2019).

25. Auyeung, E. et al. DNA-mediated nanoparticle crystallization into Wulff polyhedra. Nature 505, 73-77 (2014).

26. Boles, M. A., Engel, M. \& Talapin, D. V. Self-assembly of colloidal nanocrystals: From intricate structures to functional materials. Chem. Rev. 116, 11220-11289 (2016).

27. Singh, M., Zhou, N., Paul, D. K. \& Klabunde, K. J. IR spectral evidence of aldol condensation: Acetaldehyde adsorption over TiO2 surface. J. Catal. 260, 371-379 (2008) 
28. Park, S. J., Lazarides, A. A., Storhoff, J. J., Pesce, L. \& Mirkin, C. A. The structural characterization of oligonucleotide-modified gold nanoparticle networks formed by DNA hybridization. J. Phys. Chem. B 108, 12375-12380 (2004).

29. Gantapara, A. P., De Graaf, J., Van Roij, R. \& Dijkstra, M. Phase diagram and structural diversity of a family of truncated cubes: Degenerate close-packed structures and vacancy-rich states. Phys. Rev. Lett. 111, 1-5 (2013).

30. Ni, R., Gantapara, A. P., De Graaf, J., Van Roij, R. \& Dijkstra, M. Phase diagram of colloidal hard superballs: From cubes via spheres to octahedra. Soft Matter 8, 8826-8834 (2012).

31. Damasceno, P. F., Engel, M. \& Glotzer, S. C. Crystalline assemblies and densest packings of a family of truncated tetrahedra and the role of directional entropic forces. ACS Nano 6, 609-614 (2012).

32. Guthrie, J. P., Cooper, K. J. \& Cossar, J. The retroaldol reaction of cinnamaldehyde. Can. J. Chem. 62, 1441-1451 (1984).

33. Kline, S. R. Polymerization of rodlike micelles. Langmuir 15, 2726-2732 (1999).

34. Kim, T. H., Choi, S. M. \& Kline, S. R. Polymerized rodlike nanoparticles with controlled surface charge density. Langmuir 22, 2844-2850 (2006).

35. Umar, A., Kim, J. \& Choi, S. M. One Pot Synthesis of Monodisperse Single-Crystalline Spherical Gold Nanoparticles for Universal Seeds. (submitted)

36. Niu, W. et al. Selective synthesis of single-crystalline rhombic dodecahedral, octahedral, and cubic gold nanocrystals. J. Am. Chem. Soc. 131, 697-703 (2009). 


\section{Acknowledgements}

This work was supported by the NRF grants funded by the Ministry of Science and ICT of the Korean Government (NRF-2017R1A2A1A05001425 and NRF-2017M2A2A6A01021366) and the KUSTAR-KAIST Institute, KAIST, Korea. The authors thank the Pohang Accelerator Laboratory for providing access to the beamline $4 \mathrm{C}$ used in this study.

\section{Author Contributions}

J. K. and J. D. contributed equally to this work. J.K., J.D. and S.-M.C. conceived the study. J. K. and J. D. designed the experiments. J. K., J.D., A.U. and S.-J.L. performed the experiments. J. K. and J.-M.H. analyzed the SAXS data. All authors discussed the results. J. K. J. D. and S.M.C. wrote the manuscript.

\section{Supplementary information}

accompanies this paper at http://www.nature.com/naturecommunications

\section{Competing financial interests}

The authors declare no competing financial interests. 


\section{Figures}

(a)
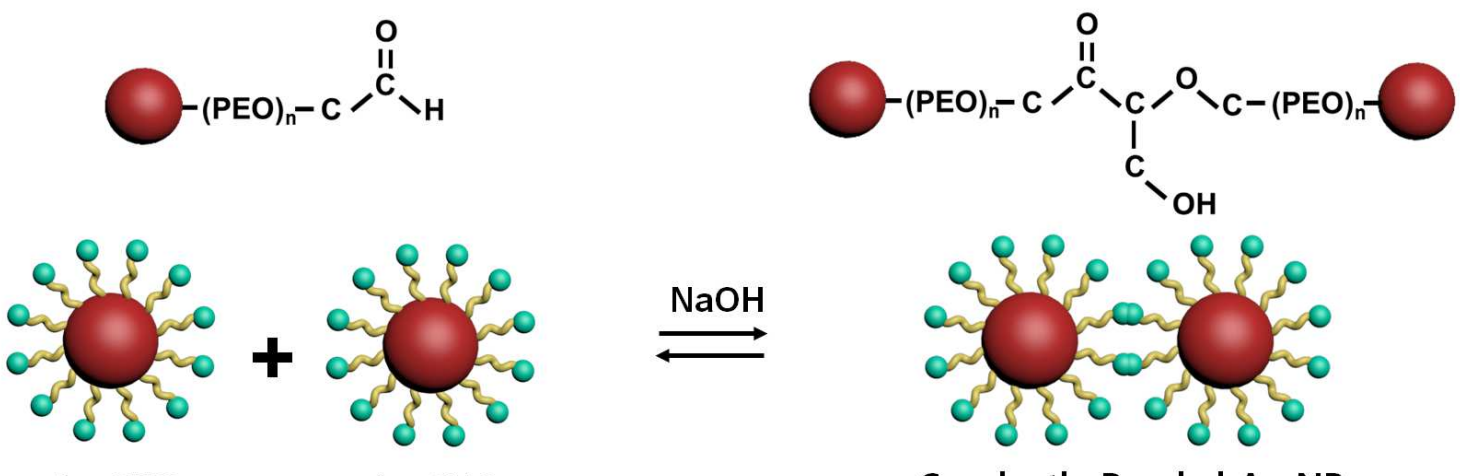

(b)

$\mathrm{Au}-\mathrm{CHO}$

$\mathrm{Au}-\mathrm{CHO}$
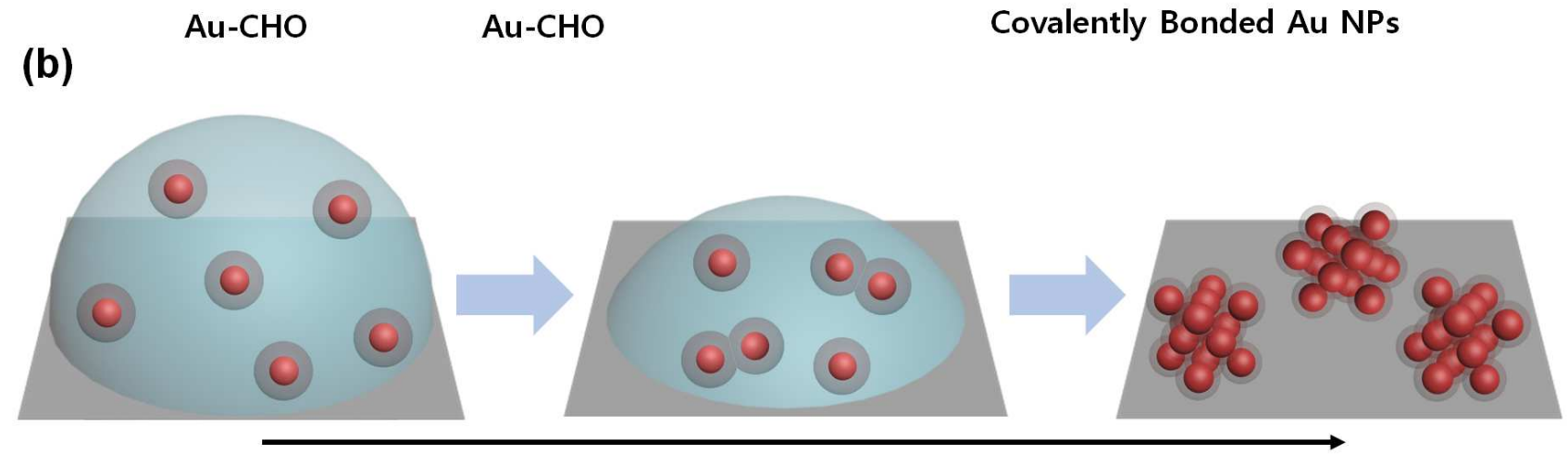

Covalent bonding \& Solvent evaporation

Figure 1

(a) Schematics for aldol reaction between formyl groups functionalized on Au nanoparticles (Au-CHO) in the presence of $\mathrm{NaOH}$. (b) Schematic for the formation of NPSCs by using covalent bonds interaction in conjunction with slow solvent evaporation. 


\section{With $\mathrm{NaOH}$}
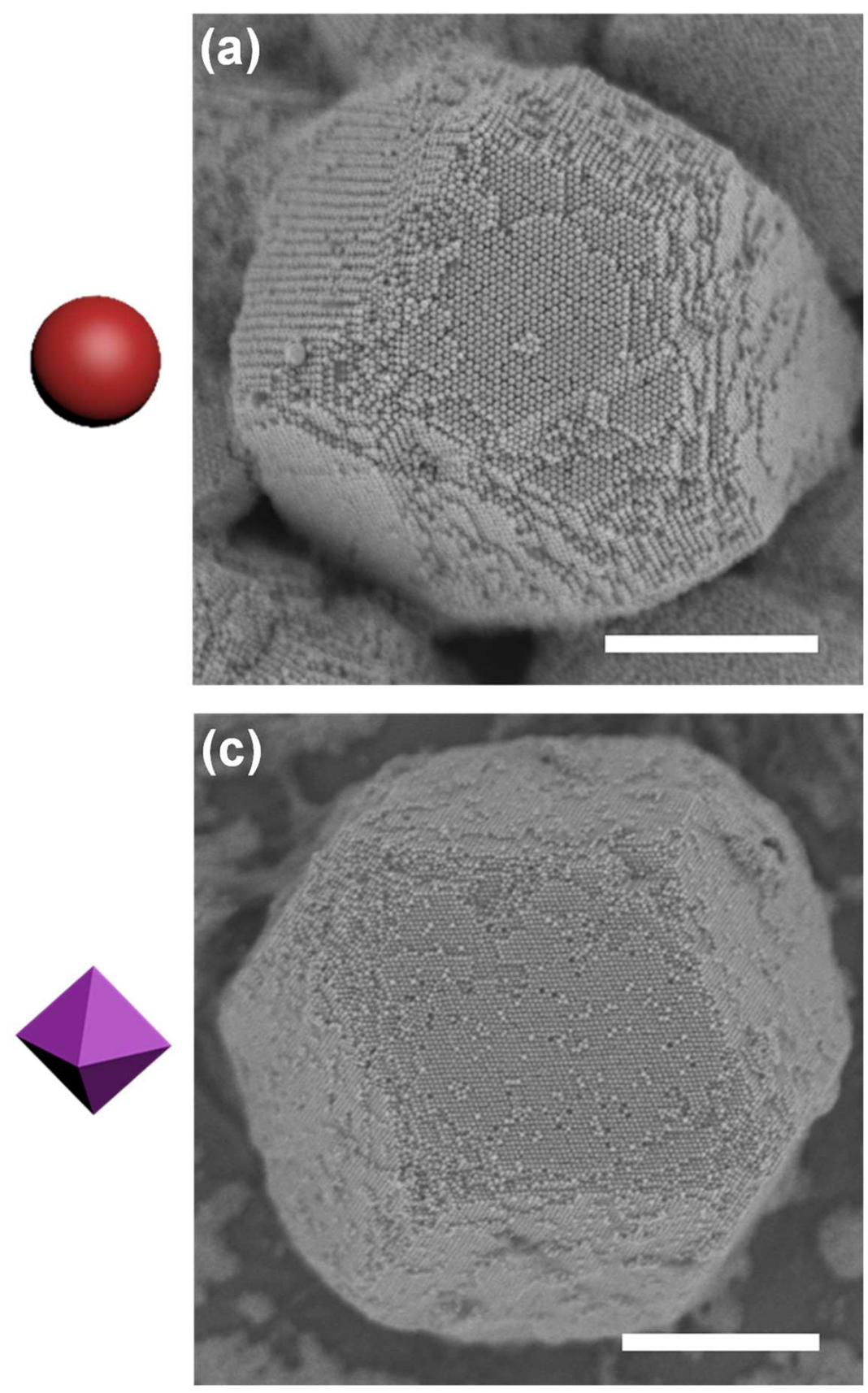

\section{Without $\mathrm{NaOH}$}
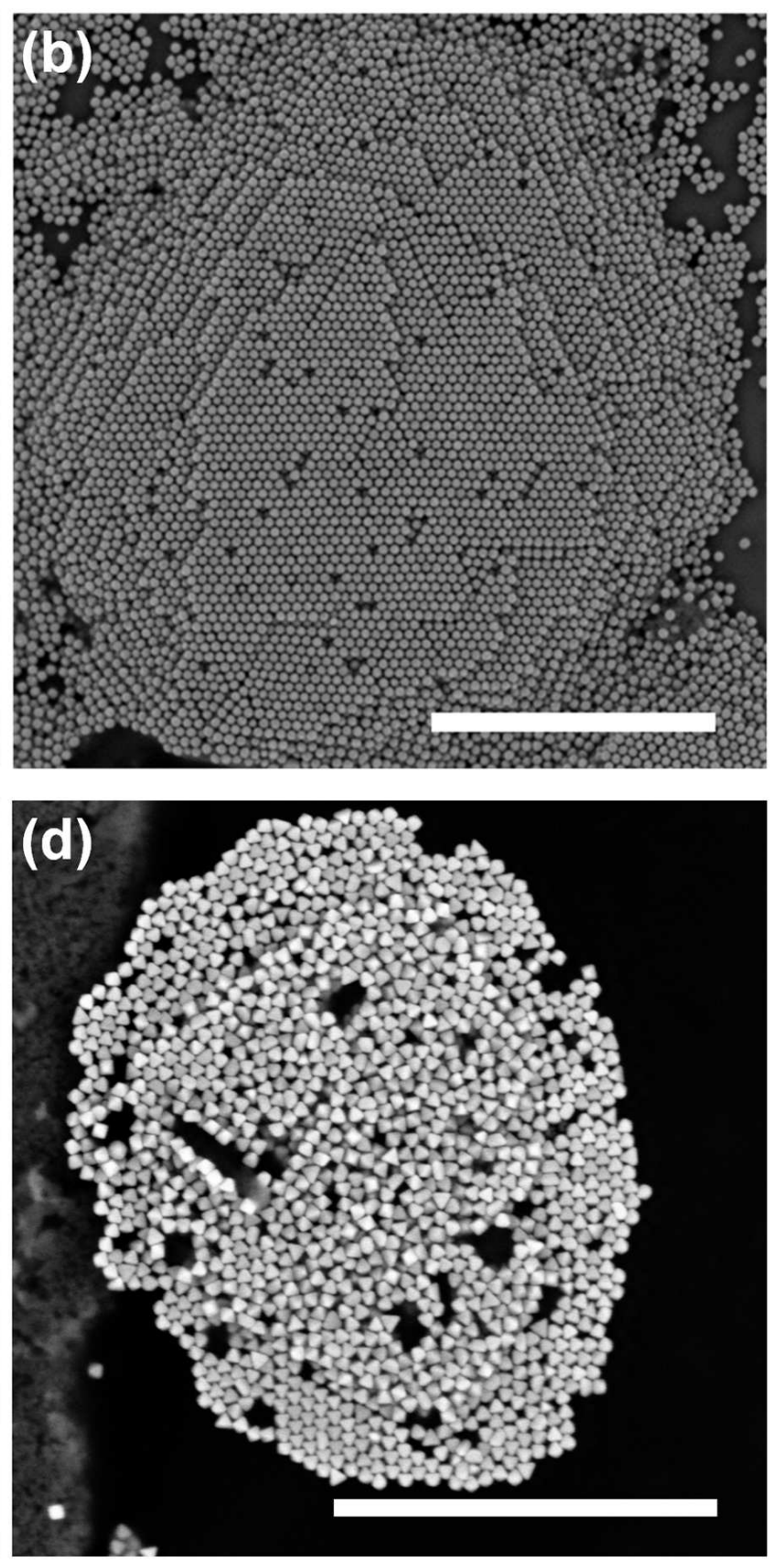

Figure 2

SEM images of NPSCs formed by spherical $(a, b)$ and octahedral $(c, d)$ Au NPs with $(a, c)$ and without (b, d) $\mathrm{NaOH}$. The scale bars are $1 \mu \mathrm{m}$ 
(a)

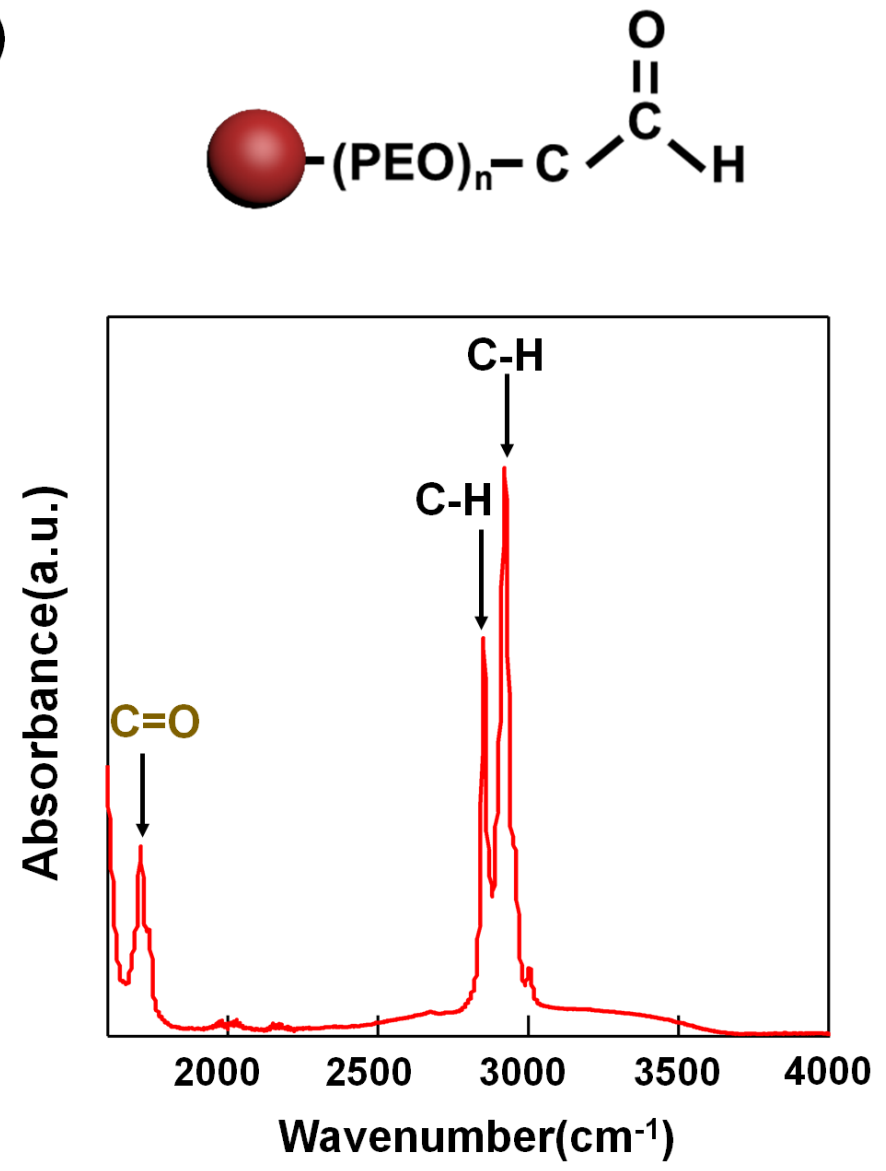

(b)
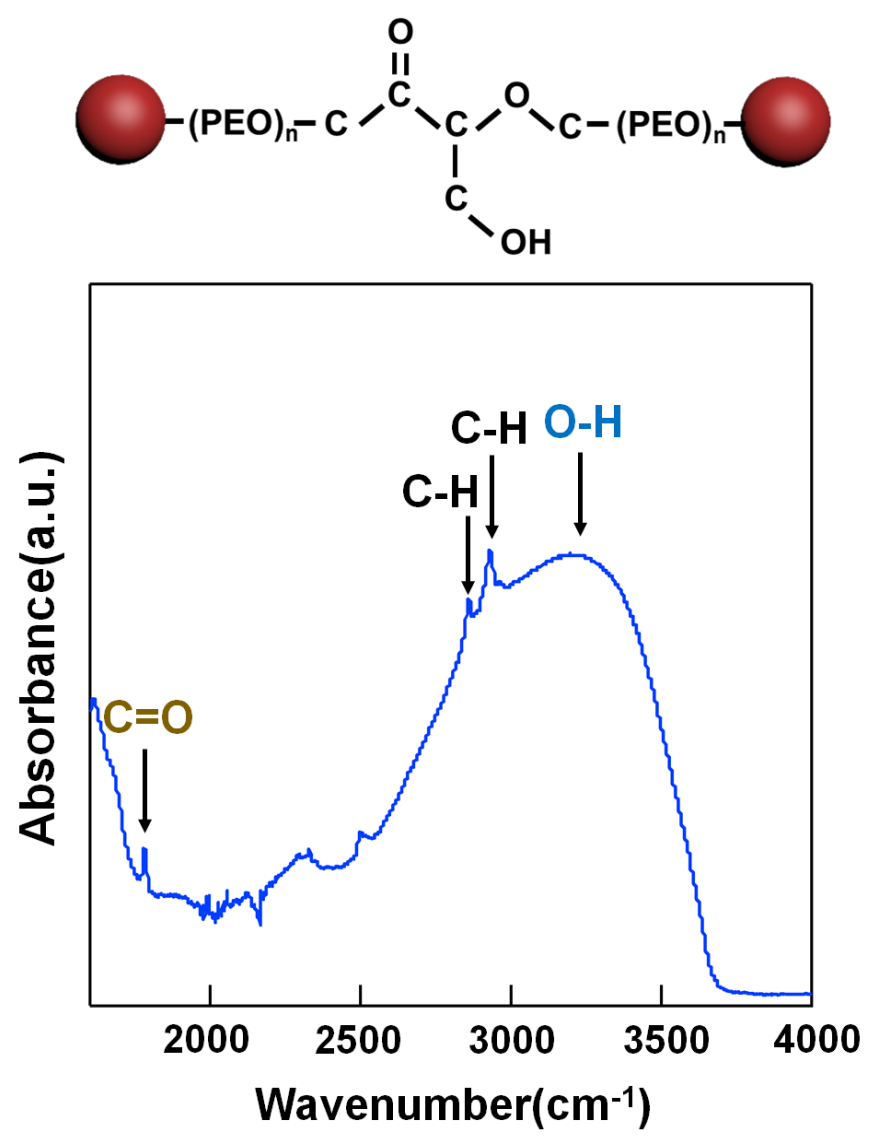

Figure 3

(a) FT-IR spectrum of spherical Au NP functionalized with HS-PEG-CHO and (b) FT-IR spectrum of spherical Au NPSCs fabricated by using aldol reaction in conjunction with slow solvent evaporation. 


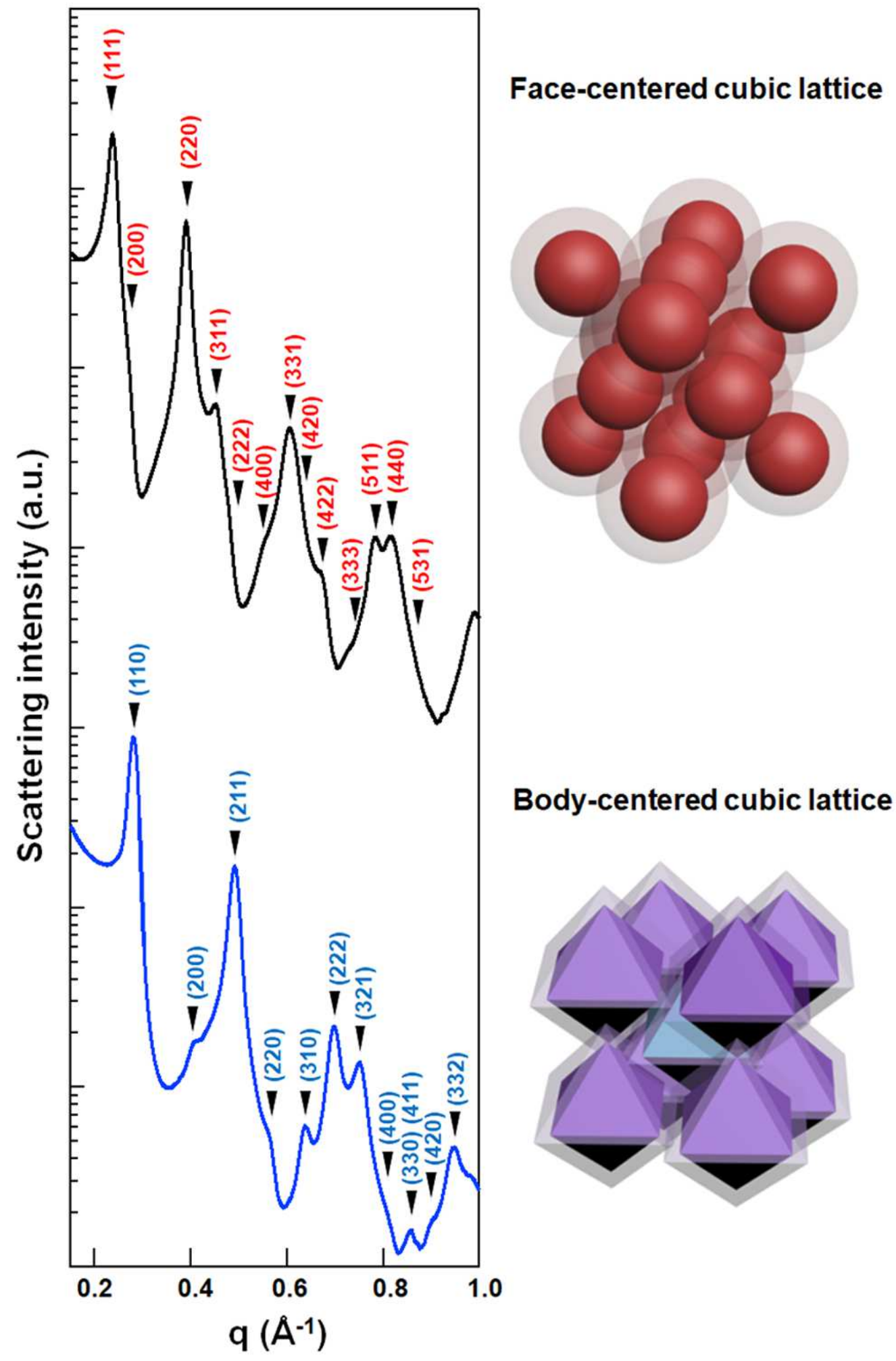

Figure 4

SAXS intensities of spherical (black) and octahedral (blue) Au NPSCs formed by aldol reaction in conjunction with slow solvent evaporation. SAXS intensity of spherical Au NPSCs is indexed with the fcc symmetry and SAXS intensity of octahedral Au NPSCs is indexed with the bcc symmetry. SAXS intensities are shifted vertically for visual clarity 

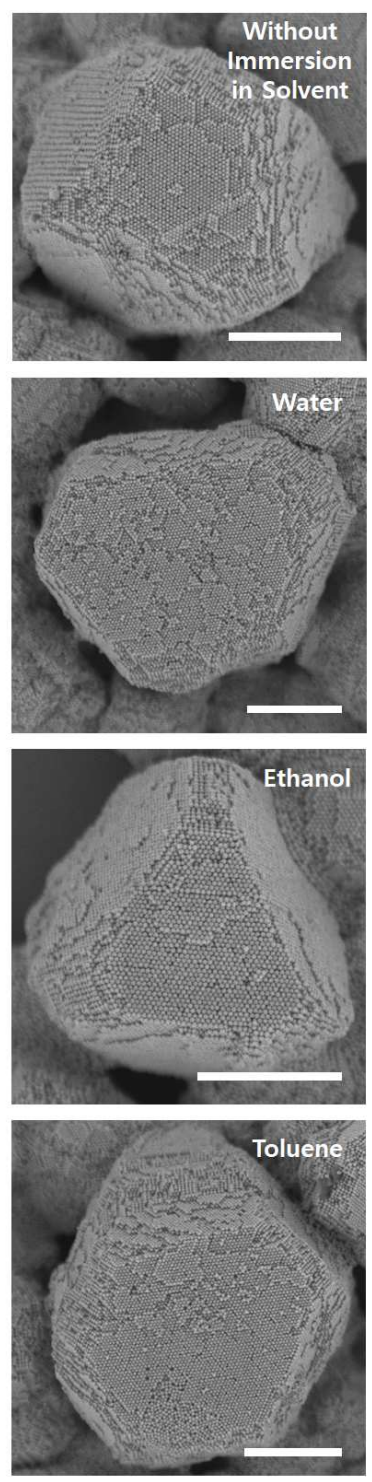
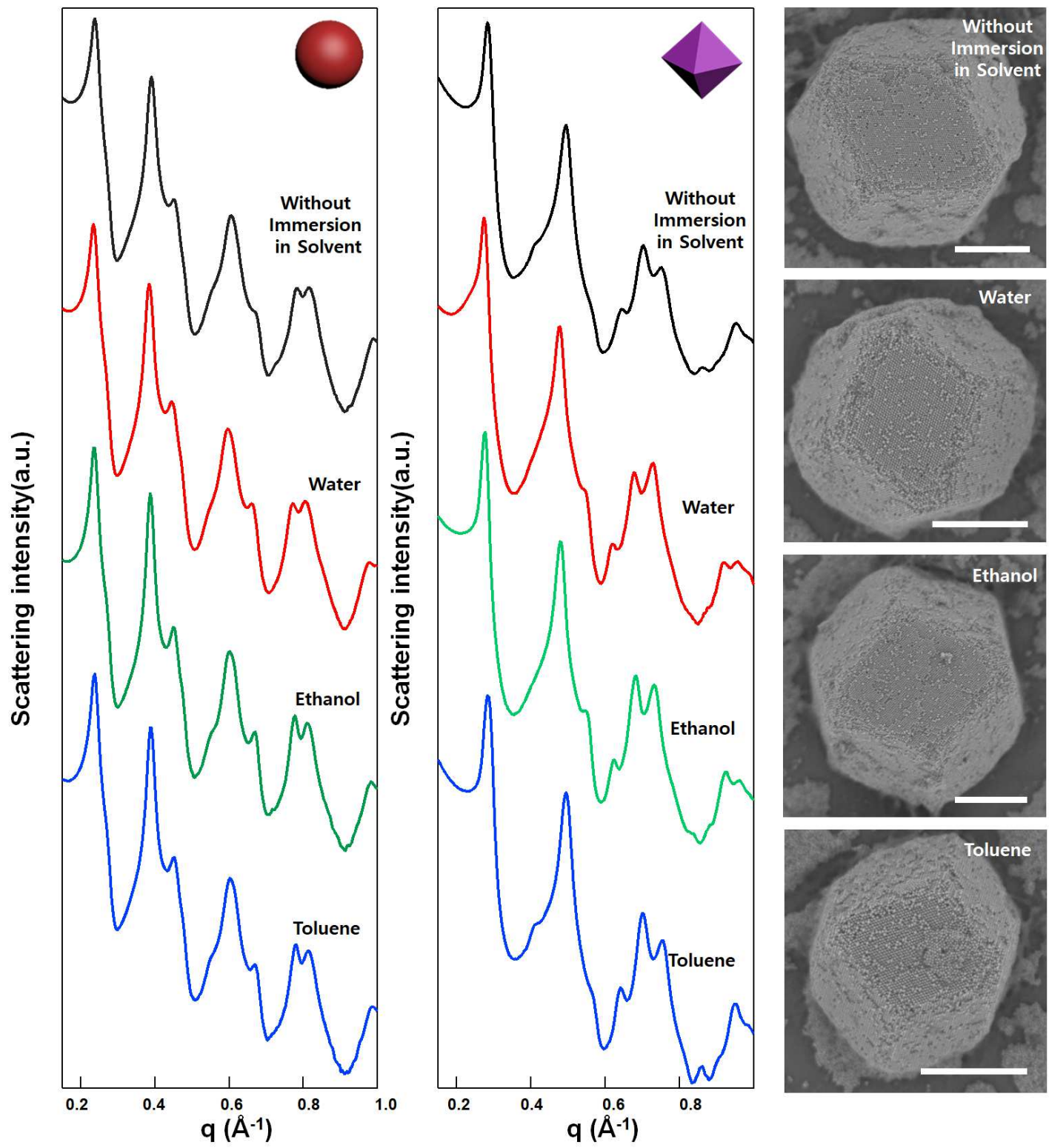

Figure 5

SAXS intensities and SEM images of spherical (left) and octahedral (right) Au NPSCs formed by aldol reaction after 24 hours of immersion in water, ethanol, and toluene. SAXS intensities and SEM images of Au NPSCs without immersion in solvents are also presented for comparison. SAXS intensities are shifted vertically for visual clarity and the scale bars in SEM images are $1 \mu \mathrm{m}$.

\section{Supplementary Files}

This is a list of supplementary files associated with this preprint. Click to download.

- NatureCommunicationsSI.pdf 